\title{
Systematic literature review on the use of social media and engagement strategies used by NHS trusts and USA healthcare systems
}

Authors: Rosie Bhogal, Sonika Sethi, Sharukh Zuberi, Nishma Gokani, Shaneil Tanna, Susana Luengo and Jagvir Grewal

\begin{abstract}
Aims
To conduct a comprehensive systematic literature review to assess how social media is currently used in the NHS and NHS engagement strategies, and compare to the USA's use.

\section{Methods}

Literature searches were performed in PubMed, MEDLINE, EBSCO and EMBASE between 2004 and 2017. Four separate search strings were created to cover both social media use in the NHS and USA, and engagement strategies in the NHS and USA. The papers that were included were critically appraised using the preferred reporting items for systematic reviews and meta-analyses statement.
\end{abstract}

\section{Results}

The search strings yielded 7,422 papers in total. After screening by initial limits, de-duplication, screening by title, abstract and inclusion/exclusion criteria, a total of 121 papers were reached. These were all critically appraised and were divided based on the theme they covered.

\section{Conclusion}

Results show there has been a rapid shift in the USA towards the uptake of social media. However, in the NHS there is limited use of social media due to uncertainty on its effectiveness and a lack of clear guidelines. Engagement strategies in the NHS have mostly taken the form of awareness campaigns and still have far to go before achieving full implementation.

\section{Conflict of interest statement}

None declared. 